\title{
Isoenzimas de fosfatasa alcalina en el suero de pacientes con insuficiencia renal
}

\author{
Ma . R. SÁNCHEZ NAVARRO, Ma . E. FERNÁNDEZ-CONDE, S. BLANCO MARTÍN, \\ C. SAMANIEGO
}

Departamento de Bioquímica. Hospital Universitario San Cecilio. Granada

\author{
ALKALINE PHOSPHATASE ISOENZYMES IN THE SERUM OF \\ PATIENTS WITH RENAL INSUFFICIENCY
}

\begin{abstract}
RESUMEN
Objetivo: estudiar la utilidad clínica de la determinación sérica de las isoenzimas de fosfatasa alcalina en pacientes con insuficiencia renal.

Material y métodos: se midieron las isoenzimas de fosfatasa alcalina en un grupo de 58 pacientes: 22 con insuficiencia renal aguda (IRA) y 36 con fallo renal crónico (IRC) y sometidos a hemodiálisis, comparándose los resultados con los de una población de 30 adultos sanos. Las isoenzimas intestinal, ósea, hepática, macromolecular e intestinal variante se separaron por electroforesis sobre gel de agarosa, cuantificándose por densitometría.

Resultados: la actividad total de fosfatasa alcalina se mostró significativamente aumentada en ambos grupos patológicos ( $\mathrm{p}<0,05$ en IRA y $\mathrm{p}<0,01$ en IRC). Los perfiles isoenzimáticos mostraron: un incremento de la fracción ósea ( $\mathrm{p}<0,05$ en IRA y p<0,001 en IRC), descenso de la fracción hepática $(\mathrm{p}<0,001)$ en ambos grupos, fracción macromolecular aumentada en pacientes con IRA $(\mathrm{p}<0,05)$ y un aumento significativo de la fracción intestinal e intestinal variante en pacientes con IRC $(\mathrm{p}<0,001)$.

Conclusión: la insuficiencia renal modifica la distribución sérica de isoenzimas de fosfatasa alcalina, su cuantificación podría servir de marcador, no invasivo, para valorar las complicaciones óseas e intestinales asociadas al fallo renal.
\end{abstract}

PALABRAS CLAVE: Insuficiencia renal aguda. Insuficiencia renal crónica. Isoenzimas fosfatasa alcalina. Osteodistrofia renal.
ABSTRACT
Objective: The aim of this study was to test the utility of serum alka line phosphatase isoenzymes determination from patients with renal insufficiency.

Material and methods: serum levels of alkaline phosphatase isoenzy mes were determined in a group of 58 patients: 22 of them suffering acu te renal insufficiency (ARI) and 36 with chronic renal failure (CRF) undergoing regular hemodialysis, results obtained were compared from a population of 30 healthy adults. Intestinal, bone, liver, macromolecu lar and intestinal variant isoenzymes, were separated by electrophore sis on agarose gel and quantified using a densitometer.

Results: were found a significant increase the total alkaline phosp hatase activity in both pathologic groups ( $p<0.05$ in ARI and $p<0.01$ in $C R F)$. Isoenzymatic profiles showed: increase of the bone fraction ( $p<0.05$ in ARI and $p<0.001$ in CRF), decrease of the liver isoenzyme $(p<0.001)$ in both groups, macromolecular fraction elevated in acute patients $(p<0.05)$ and a significant increase of the intestinal and intesti nal variant isoenzyme in the chronic patients $(p<0.001)$.

Conclusion: the renal insufficiency modified the normal distribution of alkaline phosphatase isoenzymes and the study of their serum levels could be an effective non-invasive marker, for the evaluation of bone disease and intestinal disorders associated with renal failure.

KEY WORDS: Acute renal insufficiency. Chronic renal insufficiency. Alkaline phosphatase isoenzymes. Renal osteodystrophy.

Sánchez Navarro $M^{a} R$, Fernández-Conde $M^{a} E$, Blanco Martín S, Samaniego C. Isoenzimas de fosfatasa alcalina en el suero de pacientes con insuficiencia renal. An Med Interna (Madrid) 2002; 19: 449-452.

\section{INTRODUCCIÓN}

En la denominación general de osteodistrofia renal se incluyen las complejas anomalías óseas asociadas con la enfermedad renal. Aunque los síntomas clínicos de enfermedad ósea son tardíos e inespecíficos, estudios recientes indican que las alteraciones esqueléticas comienzan en etapas tempranas de la insuficiencia renal crónica (IRC) (1-3). Si bien, el diagnóstico del tipo de osteodistrofia requiere una biopsia ósea, se ha tratado de hallar distintos marcadores bioquímicos capaces de valorar pre- cozmente, de una manera no invasiva, el alcance de las lesiones. De los marcadores disponibles, la PTH sérica junto con la fosfatasa alcalina ósea son los que mayor información aportan en el estudio de la función osteoblástica (4-8).

Otra causa de morbilidad en el enfermo renal son las alteraciones gastrointestinales. Los síntomas digestivos son frecuentes y precoces en la uremia y se sabe que la enfermedad ulcerosa péptica se presenta hasta en el $25 \%$ de los sujetos urémicos, aunque se desconoce si esta alta incidencia está relacionada con el hiperparatiroidismo secundario (9-11).

Trabajo aceptado: 21 de mayo de 2002

Correspondencia: Mª . Rosa Sánchez Navarro. Dpto. de Bioquímica. Facultad de Medicina. Av. de Madrid 18012. Granada. e-mail: rsanchez@hsc.sas.juntaandalucia.es. 
La fosfatasa alcalina (FA) es una enzima asociada con todas las células del organismo y ligada a la membrana celular. La actividad plasmática de FA es el resultado de la suma de las actividades plasmáticas de sus diferentes isoenzimas procedentes del hígado, intestino, hueso, riñón... ampliamente estudiadas y fácilmente identificables por electroforesis (12-16).

El propósito de nuestro estudio es, conocer si la enfermedad renal modifica el perfil normal de isoenzimas séricas de fosfatasa alcalina y analizar la utilidad clínica del estudio de estas modificaciones en la valoración de las manifestaciones óseas e intestinales asociadas con la disfunción renal.

\section{MATERIAL Y MÉTODOS}

Pacientes: las isoenzimas de la fosfatasa alcalina se estudiaron en un total de 88 individuos clasificados en dos grupos: un grupo formado por 30 adultos (16 mujeres y 14 varones; media de edad 45 años; rango 24-65), aparentemente sanos, que se utilizaron como control y un grupo patológico formado por 58 pacientes, que fueron seleccionados utilizando parámetros clínicos, bioquímicos y valoración objetiva de la enfermedad mediante pruebas complementarias adecuadas, compatibles con una insuficiencia renal; 22 pacientes (10 mujeres y 12 hombres; media de edad 46 años; rango 17-72) con signos y síntomas de insuficiencia renal aguda (IRA) y 36 pacientes (16 mujeres y 20 hombres; media de edad 48 años; rango 2674) con insuficiencia renal crónica (IRC) y sometidos a hemodiálisis (tiempo de diálisis 1-4 años).

Muestras: las muestras de suero se obtuvieron siguiendo el proceso habitual del laboratorio. Cuando fue posible se procesaron inmediatamente, cuando no, se mantuvieron a $-20^{\circ} \mathrm{C}$ evitándose rigurosamente las descongelaciones repetidas. En los pacientes crónicos la extracción de la muestra tuvo lugar antes de la diálisis.

Parámetros bioquímicos: urea, creatinina, calcio, fósforo y actividad total de fosfatasa alcalina se midieron a $37^{\circ} \mathrm{C}$, en Autoanalizador Hitachi 917 con reactivos Boehringer Mannheim (Automated Analysis for BM/Hitachi 917; Mannheim, F.R.G.). La separación de isoenzimas de FA: intestinal (FAI), ósea (FAO), hepática (FAH), macromolecular (FAMM) e intestinal variante (FAIV) se realizó con el método Paragon/Beckman, utilizando kits Isopal Plus (Analis, Namur, Belgium). Se utilizó gel de agarosa tamponado en buffer Tris/Borato, pH 9,45. La electroforesis tuvo lugar a 150 voltios durante 25 minutos; una vez terminada, se incubaron los geles con un substrato específico para la enzima. La cantidad relativa de las bandas se cuantificó a 600 nanometros en un densitómetro Appraise de Beckman (Beckman Instruments, Inc) y la cantidad de cada isoenzima se calculó como porcentaje de la actividad enzimática total.

Métodos complementarios: para la diferenciación y confirmación de las diferentes fracciones se utilizó metodología complementaria previa a la electroforesis. Todas las muestras se incubaron con neuraminidasa Isopal (Beckman $\mathrm{P} / \mathrm{N}$ 446145), (100 mL de suero $+20 \mathrm{~mL}$ de neuraminidasa: 30 minutos a $37^{\circ} \mathrm{C}$ ). La incubación con ficina Isopal (Beckman $\mathrm{P} / \mathrm{N}$ 446150) se utilizó para confirmar/descartar fracciones enlazadas a proteínas, la termodesnaturalización a $56^{\circ}$ durante 10 minutos y la precipitación con lecitina del germen de trigo (Lecitin WGA, Boehringer Mannheim, 1065769) para confirmar y/o diferenciar la fracción ósea y macromolecular $(1,2)$ y la incubación con antisuero antiplacentario policlonal Isopal (Beckman P/N 446140) para la confirmación de las fracciones intestinal e intestinal variante.

Análisis estadístico: las variables cuantitativas se expresan en función de su media y desviación estándar $(x \pm D E)$. Se utiliza el test paramétrico de la "t" de Student para la comparación de muestras independientes, estableciéndose el nivel de significación en un valor de $\mathrm{p}<0,05$. Los datos se procesaron con el paquete estadístico Rsigma para PC (17). La bibliografía se obtuvo gracias al Servidor de la hemeroteca de la Facultad de Medicina de Granada, utilizando el método on-line y recuperando las publicaciones sobre el tema incluidas en el sistema internacional MEDLINE desde los años 1975 a 2001.

\section{RESULTADOS}

Encontramos una actividad total de FA elevada en el suero de los enfermos con insuficiencia renal aguda y crónica en comparación con el grupo control, mientras que, la electroforesis de isoenzimas muestra un perfil característico e independiente de los valores de actividad total para cada grupo estudiado.

En el grupo control los niveles de urea y creatinina son inferiores a $50 \mathrm{mg} / \mathrm{dl}$ y $1 \mathrm{mg} / \mathrm{dl}$ respectivamente; calcio $(9,8 \pm$ $0,63 \mathrm{mg} / \mathrm{dl})$, fósforo $(3,7 \pm 0,5 \mathrm{mg} / \mathrm{dl})$ y la actividad total de FA plasmática ( $190 \pm 45 \mathrm{UI} / \mathrm{L})$. La tabla I muestra los valores séricos de los parámetros bioquímicos estudiados en los 2 grupos patológicos.

En el perfil isoenzimático del grupo control se identifican cuatro fracciones: una isoenzima hepática como forma predominante, con un valor medio del $65 \%$ de la actividad total; una isoenzima ósea; una isoenzima intestinal y una fracción macromolecular. La tabla II muestra los valores porcentuales medios y la desviación estándar (DE) de la actividad de las isoenzimas de FA encontrados en el suero de todos los grupos estudiados.

En nuestro estudio, los pacientes con insuficiencia renal aguda presentan una actividad sérica de fosfatasa alcalina $(238 \pm 91 \mathrm{U} / \mathrm{L})$, significativamente elevada con respecto al grupo control $(\mathrm{p}<0,05)$. La electroforesis de isoenzimas de FA muestra un perfil isoenzimático caracterizado por: valores de isoenzima intestinal normales $(<3 \%$ de la actividad total $)$, isoenzima ósea aumentada $(\mathrm{p}<0,05)$, isoenzima hepática disminuida $(\mathrm{p}<0,05)$ y una fracción macromolecular aumentada $(\mathrm{p}<0,05)$ con relación al grupo control.

Los pacientes con insuficiencia renal crónica muestran una actividad total de FA $(261 \pm 121 \mathrm{U} / \mathrm{L})$, significativamente aumentada con respecto al grupo control $(\mathrm{p}<0,01)$ y un perfil isoenzimático caracterizado por: un aumento significativo de los valores porcentuales de las isoenzimas intestinal y ósea $(\mathrm{p}<0,001)$, disminución de los valores de isoenzima hepática

\section{TABLA I}

PARÁM ETROS BIO Q UÍM ICOS ESTUDIADOS EN LOS GRUPOS PATO LO LÓ GICOS. VALORES MEDIOS \pm DE

\begin{tabular}{ccccc}
\hline & $\begin{array}{c}\text { Urea } \\
(\mathrm{mg} / \mathrm{dl})\end{array}$ & $\begin{array}{c}\text { Creatinina } \\
(\mathrm{mg} / \mathrm{dl})\end{array}$ & $\begin{array}{c}\text { Calcio } \\
(\mathrm{mg} / \mathrm{dl})\end{array}$ & $\begin{array}{c}\text { Fósforo } \\
(\mathrm{mg} / \mathrm{dl})\end{array}$ \\
\hline IRA & $194 \pm 49$ & $6 \pm 2,9$ & $8,9 \pm 0,8$ & $6,1 \pm 1,7$ \\
IRC & $125,5 \pm 38$ & $8,7 \pm 2,7$ & $9,2 \pm 1,1$ & $5,6 \pm 1,8$ \\
\hline
\end{tabular}


TABLA II

ISO ENZIM AS DE FO SFATASA ALCALINA. VALO RES PO RCENTUALES M EDIOS \pm DE

\begin{tabular}{lcccccc}
\hline & $\mathrm{n}$ & Intestinal & Ó sea & Hepática & M M & I.Variante \\
\hline IRA & 22 & $2,2 \pm 4,1$ & $40,3 \pm 10,8^{*}$ & $52,3 \pm 17^{*}$ & $5,2 \pm 8,3^{*}$ & - \\
IRC & 36 & $10 \pm 8,9^{* *}$ & $50,1 \pm 20,1^{* *}$ & $34,7 \pm 16^{* *}$ & $1,2 \pm 2,5$ & $3,7 \pm 1,7^{* *}$ \\
Control & 30 & $1,3 \pm 1,8$ & $31,2 \pm 10,2$ & $65,8 \pm 11,9$ & $1,1 \pm 1,7$ & - \\
\hline${ }^{*}$ p $00,05 * * 0<0,001$ & & & &
\end{tabular}

$* p<0,05 ; * * p<0,001$.

$(\mathrm{p}<0,001)$, sin cambios significativos en la fracción macromolecular y la presencia de una isoenzima intestinal variante ausente en el grupo control ( $\mathrm{p}<0,001)$.

La figura1 refleja la comparación de resultados entre los dos grupos de pacientes y el grupo control.

\section{DISCUSIÓN}

Las alteraciones óseas y gastrointestinales son complicaciones conocidas e importantes causas de morbilidad en el enfermo renal (18-21). En este estudio se mide la actividad enzimática de la fosfatasa alcalina y la distribución de sus isoenzimas en el suero de pacientes con insuficiencia renal, clasificándose los pacientes en agudos y crónicos siguiendo criterios de: historia clínica, evolución hospitalaria, exploración física, pruebas de laboratorio y técnicas adecuadas de imagen renal.

La actividad de fosfatasa alcalina sérica se tiene como un buen marcador, tanto del estímulo como de la supresión, de la actividad osteoblástica y la medida específica de FA ósea se considera mejor, que la simple medición de la actividad total para evaluar el metabolismo óseo. La FA ósea junto con la parathormona sérica se utilizan, habitualmente, en la práctica clínica, como marcadores bioquímicos del grado de actividad del hueso para separar las formas de osteodistrofia de alto y bajo remodelado en los pacientes tratados con diálisis y síntomas de enfermedad ósea (22-25).

La fosfatasa alcalina está presente en el suero bajo formas isoenzimáticas diferentes ampliamente estudiadas. Hemos utilizado un método de separación electroforética para medir la actividad de las diferentes isoenzimas de FA. Cinco isoenzi-

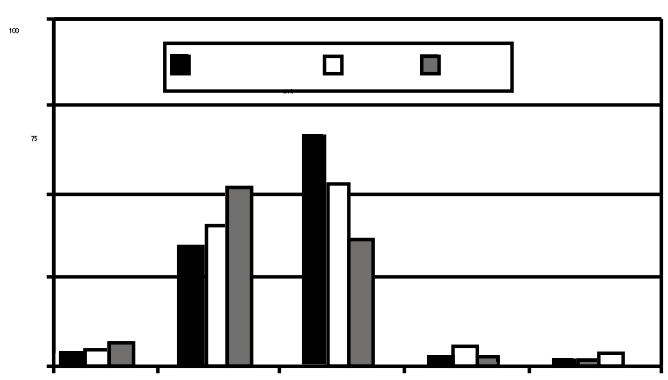

Fig. 1. Distribución de isoenzimas de fosfatasa alcalina en los pacientes renales, agudos y crónicos y comparación de resultados con el grupo control. mas, intestinal, ósea, hepática, macromolecular e intestinal variante han sido separadas y cuantificadas en el suero de los pacientes objeto del estudio.

La utilidad de las isoenzimas de FA ha sido evaluada, por varios autores, en el enfermo con fallo renal. Skillen A, y cosl. en 1977, encuentran elevación de la fosfatasa alcalina intestinal sérica en el $34 \%$ de los pacientes urémicos objeto de su estudio (26). Stepan J y cols., 1984 encuentran elevación de la isoenzima intestinal en el suero de pacientes hemodializados sin hepatopatía y una correlación inversa entre la actividad enzimática y los niveles séricos de calcio. Aunque el significado no está claro, lo atribuyen a un descenso en la eliminación de la enzima como consecuencia del hiperparatiroidismo secundario (27). Alpers y cosl. en 1988 hallan una FA intestinal aumentada en el 50\% de pacientes crónicos y además en el estudio de los riñones, extraídos durante el trasplante o postmorte, una fosfatasa con actividad inmunológica idéntica a la isoenzima intestinal en la membrana de los túbulos colectores (28). Similar a lo hallado por estos autores encontramos, en la mayoría de los pacientes crónicos dializados, un aumento de los niveles séricos de isoenzima intestinal, acompañada en todos los casos, de una fracción intestinal variante y, si bien resulta difícil hacer conclusiones definitivas acerca de las causas de este incremento, las fosfatasas intestinales podrían reflejar daño de la membrana celular y resultar marcadores eficaces de las alteraciones gastrointestinales presentes en el paciente renal crónico.

Otros autores se han interesado por este tema; recientemente Tsamura et al. encuentran, utilizando electroforesis en gel de policrilamida, una isoenzima atípica, no específica de tejido, que aparece acompañada de un deterioro del aclaramiento de creatinina en pacientes con IRC $(29,30)$. En nuestro estudio encontramos una fracción de alto peso molecular elevada en el suero de todos los pacientes agudos estudiados, esta fracción no está presente o su valor resulta normal en los pacientes crónicos dializados. En condiciones normales, la forma macromolecular no se detecta o su valor porcentual es inferior al 3\% en el suero de individuos sanos; conocida, clásicamente, como isoenzima biliar por aumentar precozmente en procesos colostásicos, hoy se sabe que aumenta en otros procesos patológicos y se ha encontrado elevada en pacientes oncológicos, enfermos diabéticos, pacientes con patología broncopulmonar (31-36). No conocemos el significado de su elevación en el enfermo renal agudo pero llama la atención el hecho de su normalización en el paciente crónico dializado.

La electroforesis es una técnica habitual en el laboratorio clínico que permite de una forma rápida y eficaz la separación y cuantificación de las diferentes fracciones de la fosfatasa alcalina. La electroforesis ofrece dos importantes ventajas sobre otros métodos que utilizan anticuerpos monoclonales 
específicos y miden, únicamente, FA ósea: una, permitir una apreciación global de todas las fracciones de la fosfatasa alcalina presentes en el suero y otra, que no existen interferencias por reactividad cruzada con la isoenzima hepática, lo que ocurre, frecuentemente, en las muestras que presentan valores bajos de FA ósea y/o actividad alta de FA hepática (37).

En nuestro estudio encontramos, además de una actividad total de fosfatasa alcalina elevada en todos los enfermos estudiados, un perfil isoenzimático característico para cada grupo de pacientes. Concluimos que la enfermedad renal, tanto aguda como crónica, modifica el valor de la actividad total y el patrón normal de isoenzimas de fosfatasa alcalina, en especial en el paciente crónico, por lo que su determinación sérica podría servir de marcador eficaz en la valoración de las manifestaciones óseas y gastrointestinales del paciente urémico. Lo que permitiría pautar un tratamiento personalizado, así como protocolizar los estudios de imagen y/o histológicos a llevar a cabo para investigar el alcance de las lesiones.

\section{Bibliografía}

1. DeVita MV, Rasenas LL, Bansal N, Gleim GW, Zabetakis PM, Gardenswartz MH, Michaelis MF. Assessment of renal osteodystrophy in hemodialysis patients. Medicine 1992; 71: 284-90.

2. Pei Y, Herez G, Greenwood C, Segre G, Saiphoo C, Fenton S. Risk factors for nenal osteodystrophy: a multivariant analysis. J Bone Miner Res 1995; 10: 149-56.

3. Salem MM. Hyperparathyrodim in the hemodialysis population: a survey of 612 patients. Am J Kidney Dis 1997; 29: 862-5.

4. Rix M, Anderassen H, Eskidsen P, Langdahl B, Olgaard K. Bone mineral density and biochemical markers of bone turnover in patientes with predialysis chronic renal failure. Kidney Int 1999; 56: 1084-93.

5. Delmas PD. biochemical markers of bone turnover for clinical assessment of metabolic disease. Endocrinol Metab Clin North Am 1990; 19: 1-18.

6. Coen G, Mazzaferro S, Ballanti P, Bonucci E.PTH and bone markers of renal ostedystrophy in predialysis chronic renal failure. J Endocrinol Invest 1992; 15: 129-33.

7. Coutteneye MM, D’Haese PC, Van Hook VO, et al. Low serum levels of alkaline phosphatase of bone origin: a good marker of adynamic bone disease in hemodialysis patients. Nephrol Dial Transplant 1996; 11: 1065-72.

8. Ferreira A, Drueke TB. Biological markers in the diagnosis of the different forms of renal osteodystrophy. Am J Med Sci 2000; 32: 85-9.

9. Var C, Gultekin F, Candan F, Turkay C, Sencan M, Icagasioglu S, et al The effects of hemodialysis on duodenal and gastric mucosal changes in uremic patients. Clin Nephrol 1996; 45: 310-4.

10. Ko CW, Chang CS, Wu MJ, Chen GH. Transient impact of hemodialysis on gastric myoelectrical activity of uremic patients. Dig Dis Sci 1998; 43: 1159-64.

11. Milito G, Taccone-Gallucci M, Brancaleone C, Nardi F, Cesca D, Boffo $\mathrm{V}$, et al. The gastrointestinal tract in uremic patients on long-term hemodialysis. Kidney Int 1985; 17: 157-60.

12. Fishman WH. Alkaline phosphatase isoenzymes: recent progress. Clin Biochem 1990; 23: 99-104.

13. Simko V. Alkaline phosphatase in biology and medicine. Dig Dis Sci. 1991; 9: 189-209.

14. Moss DW. Perspectives in alkaline phosphatase research. Chem Clin. 1992; 12: 2486-92.

15. Moss DW. Release of membrane-bound enzymes from cells and the generation of isoforms. Clin Chim Acta 1994; 226: 131-42.

16. Ronsalki SB, Foo AY. Incubation with neuraminidase and affinity electrophoresis whit wheat germ lectin compared for separating and quantifying alkaline phosphatase isoenzymes in sera. Clin Chem 1985; 31: 1198-200.

17. Moreu L, Molineri LM, Fernandez E. RSIGNA. Base de datos. Bioestadistica. Madrid: Horus Hardware S.A; 1990.

18. Ala-Kaila K, Pavonen I, Paakkala T. Increased incidence of duodenitis in chronic renal failure. Ann Clin Res 1988; 20: 154-7.

19. Cannata JB, Gómez Alonso, Grosso S, Altadill A, Díaz López JB. Osteodistrofia renal; clasificación y concepto actual. 1995; 15: 20-4.

20. Sherrard DJ, Heercz G, Pei, et al. The spectrum of bone disease in endstage renal failure. An evolving disorder. Kidney Int 1993; 43: 436-42.

21. Fletcher S, Jimes RG, Rayner HC, Harnden P, Hordon LD, Aaron JE, et al. Assessment of renal osteodystrophy in dialysis patients: use of bone mineral density and parathyroid ultrasound in comparation with bone histology. Nephron 1997; 75: 412-9.

22. Ureña P, Hruby M, Ferreira A, Ang KS, Vernejoul MC. Plasma total versus bone alkaline phosphatase as markers od bone turnover in hemodialysis patients.J Am Soc Nephrol 1996; 7: 506-12.

23. Coutteneye MM, D’Haese PC, Van Hoff VO, Lemoniatou E, Goodman W, Verpooten GA, de Broe ME. Low serum levels of alkaline phosphatase of bone origin: a goog marker of adynamic bone disease in haemodialysis patientes. Nephrol Dial Transplant 1996; 11: 1065-72.

24. Jarava C, Armas JR, Palma A. Study of renal osteodystrophy by bone biopsy. Age as an indepebdent factor, Diagnostic value of bone remodeling markers. Nefrologia 2000; 20: 362-72.

25. Sánchez MC, Bajo MA, Selgas R, et al. Parathormone secretion in peritoneal dialysis patients with adynamic bone disease. Am J Kid Disease 2000; 36: 953-61.

26. Skillen AX, Pierides AM. Serum alkaline phosphatase isoenzyme patterns in patients with chronic renal failure. Clin Chim Acta 1977; 15: 339-46.

27. Stepan J, Havranek T, Jelinkova E, Strakova M, Skrha J, Pacovsky V. Metabolic implications in the elevation of serum activity of intestinal alkaline phosphatase in chronic renal failure. Experientia 1984; 40: 896-8.

28. Alpers DH, DeSchryver-Kecskemeti K, Goodwin CL, Tindira CA, Harter H, Slatopolsky E. Intestinal alkaline phosphatase in patients with chronic renal failure. Gastroenterology 1988; 94: 62-7.

29. Tsumumura M, Ueno Y, Kinouchi T, Koyama I, Komoda T. Atypical alkaline phosphatase isoenzymes in serum and urine of patients with renal failure.Clin chim Acta 2001; 312: 169-78.

30. Kuwana T, Rosalki SB. Intestinal variant alkaline phosphase in plasma in disease. Clin Chem 1990; 36: 1918-21.

31. Van Hoff VO, Van Oosterom AT, Lepotre LG, De Broe ME. Alkaline phosphatase isoenzyme patterns in malignant disease.Clin Chem. 1992; 12: 2546-51

32. Tibi L, Collier A, PatricK AW, Clarke BF, Smith AF. Plasma alkaline phosphatase isoenzymes in diabetes mellitus. Clin Chim Acta 1988: 167: 147-56.

33. Nishio, Sokuma, Nakamura, Horai, Ikegami, Matsuda. Diagnostic value of high molecular weight AP in detection of hepatic metastasis in patients with lung cancer. Cancer 1986; 57: 1815-9.

34. Capelli A, Lusuardi M, Ceruti CG, Donner CF. Lung alkaline phosphatase as a marker of fibrosis in chronic interstitial disorders. Am J Respir crit Care Med 1997; 155: 249-53.

35. Sánchez Navarro MR, Fernández-Conde ME, Oliver C, Hurtado JA, Samaniego M. Alkaline phospatase isoenzymes in the serum and bronchoalveolar lavage of patients with broncopulmonary pathology. 2000; 17: 182-5.

36. Wolf PL. Clinical significance of serum high-molecular-mass alkaline phosphatase, alkaline phosphatase.lipoprotein-X complex, and intestinal variant alkaline phosphatae. J clin Lab Anal 1994; 8: 272-6.

37. Van Hoof VO, Martin M, Blockx P, Prove A, Van Oosterom A, Couttenye MM, et al. Inmunoradiometric method and electrophoretic system comapred for quantifying bone alkaline phosphatase in serum. Clin Chem $1995 ; 41: 853-7$. 